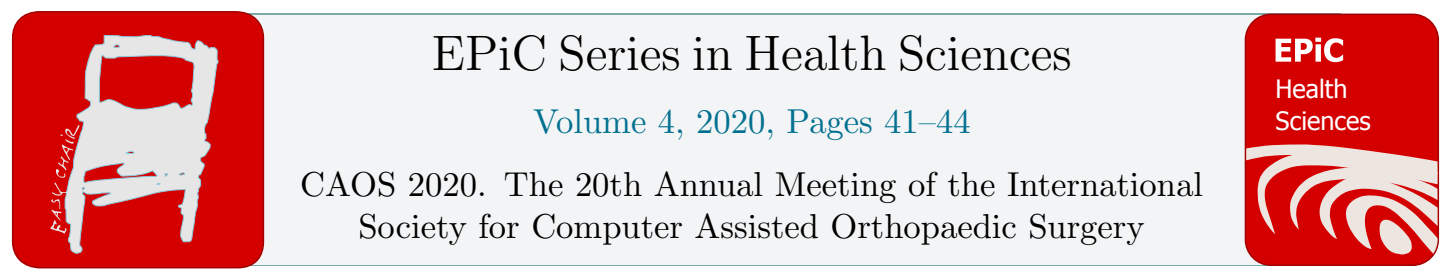

\title{
Application-Specific Learning Curve in Computer-Assisted Total Knee Arthroplasty
}

\author{
Yifei Dai ${ }^{1 *}$, Laurent Angibaud ${ }^{1}$, Guillaume Bras², Cyril Hamad ${ }^{2}$, Jefferson \\ Craig Morrison ${ }^{3 *}$ \\ ${ }^{1 *}$ Exactech Inc, Gainesville, FL, 32653, USA \\ ${ }^{2}$ Blue Ortho, Gieres FR \\ ${ }^{3 *}$ Southern Joint Replacement Institute, Tristar Centennial Hospital, Nashville, TN \\ craig@sjri.com; yifei.dai@exac.com
}

\begin{abstract}
This study employed an advanced method (CUSUM) to analyze the learning curve regarding surgical efficiency (time) using two CAOS applications, which were designed to address user needs with different levels of comprehensiveness in term of offered guidance and instrumentation requirements. Two group of surgeons, each used either CAOS applications were included in the study. The first 50 CAOS TKA cases from each surgeon were analyzed to identify the learning curve. The duration of learning, as well as the impact of learning based on surgical time, were assessed with regard to the specific CAOS application and surgeon's previous CAOS experience level. The data demonstrated differences in term of pattern of adoption during learning process between the two CAOS applications. However, the learning process was not sensitive to surgeon's experience level.
\end{abstract}

\section{Introduction}

Since the introduction of computer-assisted orthopedic system (CAOS) in total knee arthroplasty (TKA), the adoption of the system is perceived to be challenged by costs and a significant learning curve. The successful adoption of a CAOS system should demonstrate reasonable learning effort. The methodology applied in existing studies usually compared surgical time between the cases performed during learning and those from the later cases, with an assumed duration (number of cases) of the learning curve [1-2]. Researchers have performed logarithmic regression on the initial CAOS case series to find the duration of the learning phase [3]. However, as the surgical time data is often, by nature, noisy, the regression result can be difficult to evaluate.

The recent applications of cumulative sum control chart (CUSUM) in the medical field has gained interests among researchers [4]. As an effective tool for detecting the stabilization of a process 
(completion of learning), this methodology may enable objective comparison of learning curves between different orthopedic applications. The goal of this study was to leverage CUSUM for a comparative assessment of learning curve between two contemporary CAOS applications.

\section{Materials and Methods}

Two distinct TKA applications offered by a CAOS system were investigated in this study. Application A was a full-sized CAOS system with dedicated CAOS specific instrumentation, bringing comprehensive guidance throughout TKA surgical workflow and full versality based on surgeons' preferences (surgical target, approach, order, anatomical references, etc). Application B was developed to provide CAOS augmentation to the conventional mechanical instrumentation, offering streamlined guidance for the sole proximal tibial and distal femoral cuts without substantial change of instrumentation and disruption of the conventional surgical workflow.

Technical records on primary TKA cases performed using the two applications were reviewed, including data from 10 surgeons (based on their previous CAOS experience, 7 senior, 3 novice) for Application A and 4 surgeons (1 senior, 3 novice) for Application B. CUSUM analysis on surgical time were performed on the first 50 cases for each surgeon [4]. A horizontal trend in the plotted CUSUM curve signified the deviances were equally balanced around 0 , indicating the process was operating with stability. The case number (learning duration) by which the CUSUM curve entered the horizontal trend was identified as the end of learning duration for the surgeon. The learning duration was compared between the two applications, as well as between senior surgeons and the novice surgeons within each application. The impact of learning on surgical time was assessed between the cases in the learning curve group (cases within the learning duration) and the later cases (\#41-50) for each application. Significance was defined as $\mathrm{p}<0.05$.

\section{Results}

Compared to the raw surgical time graph (Fig 1A), the CUSUM plot clearly exhibited three unique phases in the first 50 cases of each surgeon, with Phase II demonstrating stabilization of the process (Fig 1B). All surgeons demonstrated stabilization of the surgical process, indicating successful completion of the learning process. The actual shape of the 3 phases differed from surgeon to surgeon, reflecting each individual's characteristics of learning. On average, it took 12-13 cases to complete the learning curve for Application A, whereas Application B required 3 cases (Table 1). The surgeons spent approximately $15 \mathrm{~min}$ and $8 \mathrm{~min}$ longer during learning (compared to cases \#41-50) for Application A and Application B, respectively. Within each CAOS application, no significant difference was found between the senior and novice surgeons regarding learning duration and time increase during leaning (N.S.) (Table 1). 

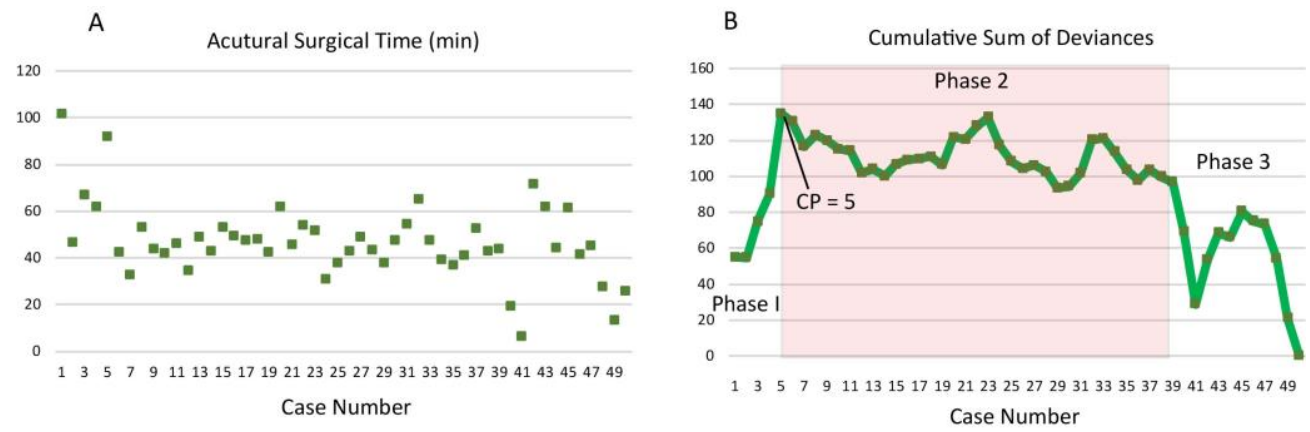

Figure 1. Graphs on the A) actual surgical time and B) CUSUM charts for a representative surgeon. The graph was plotted according to the chronological case numbers.

\begin{tabular}{lccc}
\hline & Senior Surgeon & Novice Surgeon & Pooled \\
Application A & & & \\
$\mathrm{N}$ & 7 & 3 & 10 \\
Learning Duration (\#cases) & $13.0 \pm 7.1$ & $12.0 \pm 7.5$ & $13.4 \pm 7.4$ \\
Time Increase during Learning (min)* & $13.0 \pm 8.6$ & $16.2 \pm 14.6$ & $13.3 \pm 10.7$ \\
& & & \\
Application B & 1 & 3 & 4 \\
$\mathrm{~N}$ & 3.0 & $3.0 \pm 2.6$ & $2.5 \pm 2.2$ \\
Learning Duration (\#cases) & 8.5 & $8.3 \pm 3.1$ & $8.4 \pm 2.2$ \\
Time Increase during Learning (min)* & & & \\
\hline
\end{tabular}

* Calculated as Average(learning duration) - Average(cases \#41-50)

Table 1. Summary of learning characteristics in the senior surgeons, novice surgeons, and pooled surgeon group for the two CAOS applications. No significant difference was found between senior and novice surgeons (N.S.) in learning duration and time increase.

\section{Discussion}

This study used CUSUM analysis to demonstrate application specific learning curves for two CAOS applications, each developed to address user needs with different level of comprehensiveness in CAOS guidance and instrumentation requirement (CAOS specific instrumentation or augmentation to conventional mechanical instruments). Based on surgical efficiency (time), the analysis investigated the adoption of the surgery as a process that eventually stabilizes with mastery of the task.

Regardless of the surgeon's previous CAOS experience, adopting either application did not result in substantial learning curve, with moderate (12-13 cases) learning duration for Application A and minimal learning duration ( 3 cases) for Application B. The learning duration only moderately (Application A) or minimally (Application B) increased surgical time.

Despite the CUSUM method being proposed since the 1970s for analyzing learning curve for surgical procedures [5,6], and since then being applied to various medical fields [4], the use of this method in TKA is very limited [7]. Utilization of this advanced method in this study allowed assessment of differences in learning between individual surgeons and CAOS applications. 


\section{References}

[1] Petrera P, et al. CAOS 2015

[2] Howard JL, et al. Bone Joint J 2016.98-B(Supp 20)88.

[3] Jenny JY, et al. Knee. 2008;15(2):80-4.

[4] Bokhari MB, et al. Surg Endosc. 2011;25(3):855-60.

[5] Chaput de Saintonge DM, Vere DW. Lancet. 1974;1:120-1.

[6] Wohl H. N Engl J Med. 1977;296: 1044-5.

[7] De Gori M, et al. Knee. 2017;24(3):615-21. 